H. Shangguan, L. W. Casperson, K. W. Gregory and S. A. Prahl, "Penetration of Fluorescent Particles in Gelatin During Laser Thrombolysis," Diagnostic and Therapeutic Cardiovascular Interventions VII, R. R. Anderson et al. Ed., Proc. SPIE 2970, 10-18, (1997).

\title{
Penetration of Fluorescent Particles in Gelatin During Laser Thrombolysis
}

\author{
HanQun Shangguan $^{a}$, Lee W. Casperson ${ }^{b}$, Kenton W. Gregory ${ }^{a}$ and S. A. Prahl ${ }^{a}$ \\ ${ }^{a}$ Oregon Medical Laser Center, 9205 SW Barnes Rd, Portland, OR 97225 \\ ${ }^{b}$ Department of Electrical Engineering, Portland State University, Portland, Oregon 97207
}

\begin{abstract}
The use of pulsed laser energy to clear arteries obstructed by thrombus (blood clot) and plaque has emerged as a promising method for the treatment of cardiovascular diseases such as myocardial infarction and stroke. Current techniques for laser thrombolysis are limited because they cannot completely clear the clot in arteries, especially where a large volume clot is presented. Mural clot is a potent stimulus for reocclusion. We suggest that the combination of laser thrombolysis and localized intramural delivery of clot-dissolving drugs during the procedure may be a solution to this limitation.

Ninety pulses of 30-70 mJ were delivered onto gelatin-based thrombus model with a flushing catheter. A solution of $1 \mu \mathrm{m}$ fluorescent particles as a drug model was injected at a rate of $4 \mathrm{~mL} / \mathrm{min}$ in coincidence with the laser delivery. The controls were performed by injecting drug after laser thrombolysis. We measured the penetration of the particles in gelatin and the sizes of the lumen and stained areas. The results of this study demonstrated the possibility of enhancing laser thrombolysis by delivering drugs into thrombus. It was found that the particles could be driven several hundred micron in gelatin, and the lumen areas would be increased up to $25 \%$ if the areas were dissolved by the drugs.
\end{abstract}

Keywords: photomechanical drug delivery, laser thrombolysis, laser-induced hydrodynamic pressure

\section{INTRODUCTION}

Laser thrombolysis is a promising method of clearing arteries that are obstructed by thrombus and plaque. ${ }^{1}$ It has potential advantages over bypass surgery, balloon angioplasty, and other forms of vascular intervention. Laser pulses can be delivered into arteries through fiber-optic catheters, thus avoiding major surgery. The thrombus is removed by the laser pulses rather than being displaced by them, thereby, potentially reducing the high rate of restenosis that occurs with balloon angioplasty. The laser energy is strongly absorbed by the thrombus and poorly absorbed by the adjacent tissue. Thus, selective and safe thrombolysis using laser radiation can be achieved. The use of small fiber-optic catheters offers the possibility of removing thrombus in small vessels (e.g., brain vessels) or in larger vessels (e.g., bypass grafts), in which no effective therapies are available. ${ }^{2}$ However, one concern for laser thrombolysis is the mural blood clot left in arteries after the laser thrombolysis procedure, which may be a potential limitation for this therapy. We suggest that the combination of laser thrombolysis and localized intramural delivery of clot-dissolving drugs during the procedure may be an alternative solution to this limitation, i.e., the majority of the clot can be removed by laser pulses and the mural clot is dissolved by the drugs. The present study was motivated by this concept.

Current techniques for localized drug delivery use balloon-based catheters to infuse thrombolytic agents into thrombus at an angioplasty site. The intramural deposition of therapeutic agents may provide prolonged thrombolytic action at the infusion site. ${ }^{3}$ Basically, two mechanisms are involved in these procedures: 1) displacement of thrombus due to the balloon dilation and 2) thrombolytic action by therapeutic agents. However, the primary problems with the modified balloon catheter designs are their cumbersome size and long inflation times, as well as the risk of medial injury occurring during the balloon inflation process, thus potentially limiting the benefits of local drug delivery.

Direct correspondence to SAP: prahl@ece.ogi.edu; (503) 216-2197; http://omlc.ogi.edu 
We have recently demonstrated that drug could be delivered into thrombus by use of hydrodynamic pressures following the laser-induced bubble expansion and collapse. ${ }^{4}$ The bubbles were formed at the fiber tip due to the absorption of laser energy by the surrounding absorbing liquids. The spatial distribution of photomechanically delivered drug depended on parameters such as laser energy, absorption coefficient, fiber size, and material strength. The bubble formation on the thrombus surface due to the absorption of laser energy by the thrombus could also cause flow motion near the cavitation bubble at a speed of up to $12 \mathrm{~m} / \mathrm{s} .{ }^{5}$ We hypothesize that hydrodynamic flow resulting from the bubble formation during laser thrombolysis can be used as a driving force to facilitate drug delivery. This form of localized drug delivery has been termed photomechanical drug delivery. ${ }^{6}$

Our objective in this in vitro study was twofold. First, we wished to quantitatively compare the effectiveness of thrombolysis by use of 1) laser thrombolysis and 2) photomechanical drug delivery. Such a comparison is important to clearly establish any benefit that may be associated with photomechanical drug delivery. Second, we wished to identify the mechanism of photomechanical drug delivery during laser thrombolysis through visualization of bubble formation with flash photography and measurement of laser-induced pressure waves using a PVDF transducer. Solutions of fluorescent spheres were used as a drug model that allowed us to easily visualize the sphere distribution in thrombus under fluorescence microscopy. Gelatin-based thrombus phantoms were used to avoid the complicated biological variations of real thrombus.

\section{MATERIALS AND METHODS}

\subsection{Sample Preparation}

Two types of gelatin targets were formed in $1 \mathrm{~cm}$ cuvettes. Drug delivery experiments used a uniformly absorbing gelatin made by adding Blue 15 dyes (Sigma) into 3.5\% 175 bloom liquid gelatin (Sigma) and curing in the $1 \mathrm{~cm}$ cuvettes. The blue gelatin samples had an absorption coefficient of $100 \mathrm{~cm}^{-1}$ at $577 \mathrm{~nm}$. The cured gelatin samples were carefully removed from the cuvette and cut into $\sim 5 \mathrm{~mm}$ thick sections before the experiments. The samples for flash photography were made by pouring clear gelatin into $1 \mathrm{~cm}$ cuvettes to form $2-3 \mathrm{~cm}$ thick samples with cylindrical geometries. The channels were constructed about $2 \mathrm{~mm}$ in diameter to simulate blood vessels; the normal adult human coronary artery is $2-4 \mathrm{~mm}$ in diameter. A piece of porcine clot was inserted into the channel and then was covered with distilled water at room temperature.

The solution seeded with fluorescent latex microspheres (Molecular Probes, Inc.) was used as a drug model. The microspheres consisted primarily of polystyrene chains, and they were supplied as suspensions $(2 \%$ solids with $1 \mu \mathrm{m}$ diameter) in water plus $2 \mathrm{mM}$ sodium azid. They were nonporous, hydrophobic, and photostable. The excitation and emission maxima were at $488 \mathrm{~nm}$ and $515 \mathrm{~nm}$ respectively. The concentration of the solution was about $7.5 \times 10^{7}$ particles $/ \mathrm{ml}$ made by adding $0.5 \mathrm{ml}$ of the suspensions with a concentration of $3 \times 10^{10}$ particles $/ \mathrm{ml}$ into $200 \mathrm{ml}$ of distilled water. No attempt was made to correlate this solution to any specific drugs for this study.

\subsection{Experiments}

The effectiveness of thrombolysis was evaluated by comparing the sizes of lumen created by laser ablation and stained by the fluorescent spheres. A basic assumption of this study was that the stained areas could potentially be dissolved by the delivered drug. Two experimental protocols were employed: 1) Drug was infused into the lasercreated channel after laser thrombolysis and 2) Drug was delivered into the thrombus in coincidence with the laser pulses. The drug was injected at a rate of $4 \mathrm{ml} / \mathrm{min}$ through the catheter for a similar time $(\sim 30$ seconds $)$ in the two experiments. All samples were carefully washed with clear water before being frozen and sectioned. Frozen sections were examined under light and fluorescence microscopy. The areas and penetration of the spheres in the gelatins were measured. The penetration depth was measured from the edge of the lumen to the fluorescent spheres inside the gelatin sample.

A fiber-optic flushing catheter was used for drug delivery and light delivery. This catheter consisted of a $1 \mathrm{~mm}$ Teflon tube filled with a $300 \mu \mathrm{m}$ optical fiber. The drug was infused into the thrombus through the tube connected with a syringe. The flow rate was $4 \mathrm{ml} / \mathrm{min}$ controlled by a syringe pump. Laser radiation was provided by a $1 \mu \mathrm{s}$ pulsed dye laser (Palomar Medical Technologies) emitting $577 \mathrm{~nm}$ light at $3 \mathrm{~Hz}$. The laser pulses of $30-70 \mathrm{~mJ}$ were delivered via the flushing catheter onto the thrombus under clear liquids. 


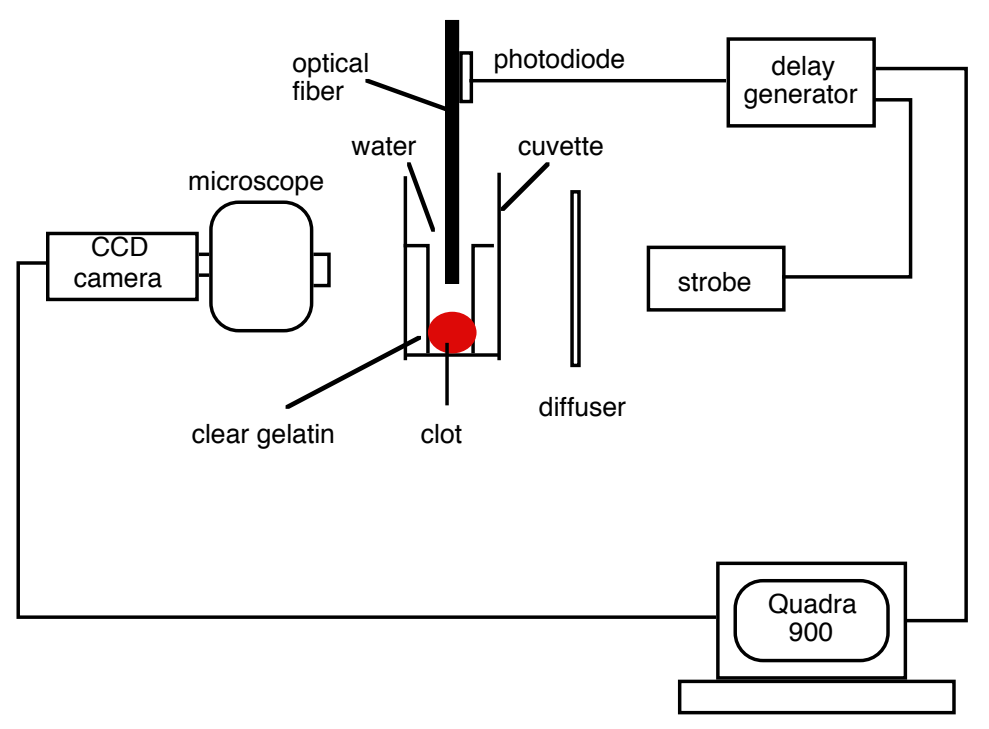

Figure 1. Schematic of experimental setup for visualizing the bubble formation on clot or gelatin.

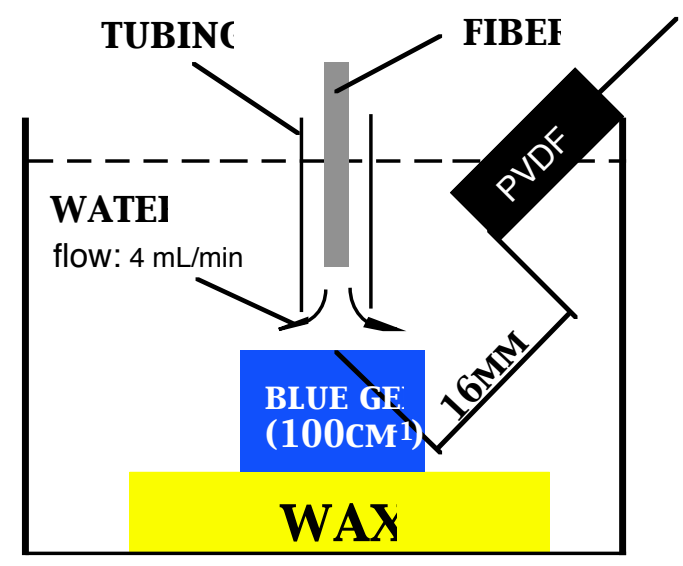

Figure 2. Schematic of apparatus for pressure measurement.

The laser interaction with porcine clot was visualized with a flash photography setup (Figure 1). Single pulses of $50 \mathrm{~mJ}$ were delivered onto porcine clot through a $300 \mu \mathrm{m}$ fiber placed $1 \mathrm{~mm}$ above the surface of the clot. The laserinduced pressure waves were measured using a PVDF transducer (KP 117, Ktech) shown in Figure 2. The transducer was positioned $16 \mathrm{~mm}$ away from the ablation site. The experiments were performed on thrombus phantoms with an absorption coefficient of $100 \mathrm{~cm}^{-1}$ at $577 \mathrm{~nm}$. The samples were immersed under water at room temperature.

\subsection{Statistical Analysis}

All data were reported as mean \pm standard deviation. The lumen size, stained area, and penetration depth of the spheres in the gelatin samples were compared. The statistical significance of differences was determined using a twotailed Student's $t$-test. An unpaired $t$-test was used to analyze the data for laser thrombolysis and photomechanical drug delivery. A value of $p<0.05$ was considered to be significant.

\section{RESULTS}

As shown in Figure 3, the areas (i.e., lumen area plus stained area) didn't increase significantly through drug delivery after laser thrombolysis, while the areas increased after the photomechanical drug delivery procedure. The 


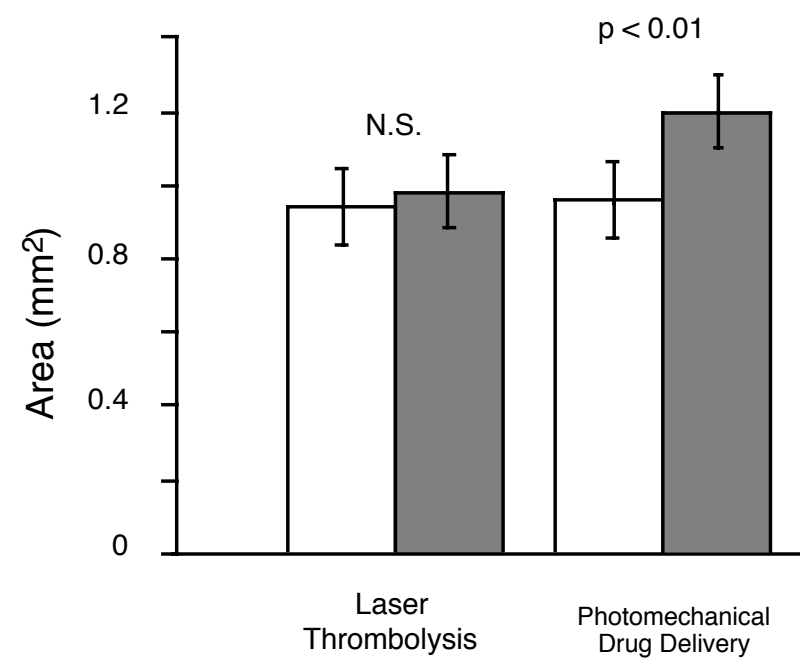

Figure 3. Comparison of lumen areas generated by two different methods: laser thrombolysis and photomechanical drug delivery. Ninety laser pulses of $50 \mathrm{~mJ}$ were delivered into gelatin samples with an absorption coefficient of $100 \mathrm{~cm}^{-1}$ for both laser thrombolysis and photomechanical drug delivery experiments. A flushing catheter with a $300 \mu \mathrm{m}$ fiber was used for the light delivery. The open markers represent the lumen area and the filled markers indicate the areas including the lumen area and the stained area. Error bars present the standard deviation of four samples.

areas increased up to $25 \%$ when we consider the areas stained by the spheres as compared with the lumen generated by laser ablation alone.

The penetration measurements revealed that the maximal penetration was not correlated with the laser energy (Figure 4). When the laser energy increased to greater than a value somewhere between $50-70 \mathrm{~mJ}$, the penetration depth decreased. However, the lumen sizes increased significantly with increasing laser energy (Figure 5). The pressure measurements also showed that the pressures increased as the laser energy increased (Figure 6) and the lumen sizes were proportional to the bubble expansion pressure (Figure 7).

Visualization of the interaction of a laser pulse with clot showed that a full size bubble was formed in the clot (Figure 8). The bubble dynamics on clots was similar to the dynamics on gelatin targets. Figure 9 shows the cavitation bubble growth and collapse produced by a $50 \mathrm{~mJ}$ laser pulse. The bright spot in the sixth frame was the flash of light from the strobe. The bubble expansion caused the dilation of the channel. The maximal dilation was reached after about $60 \mu \mathrm{s}$ and amounted to $130 \%$ of the initial channel diameter. The subsequent bubble collapse induced an invagination of the channel wall. The minimal diameter was $\sim 88 \%$ of the initial value. No oscillation of the cavitation bubble was observed. The mass ejection followed the bubble collapse. 


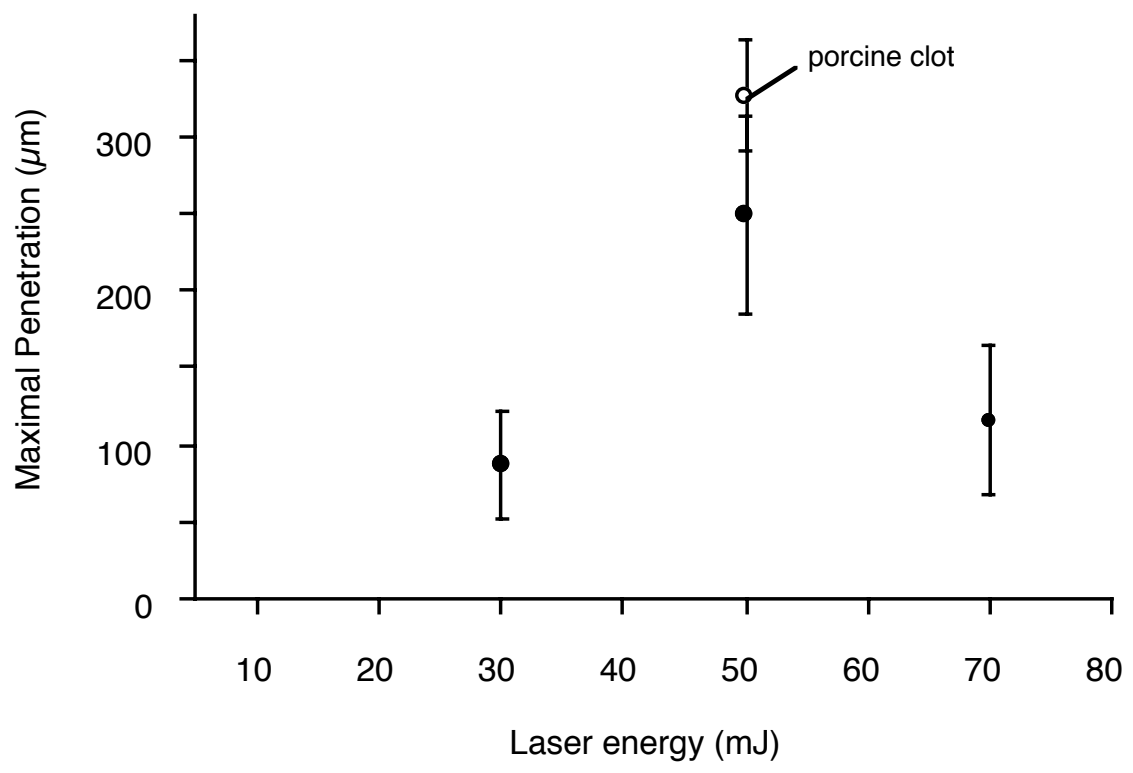

Figure 4. Maximal penetration of fluorescent spheres inside gelatin samples as a function of laser energy using photomechanical drug delivery. The laser pulses were delivered via a flushing catheter with a $300 \mu \mathrm{m}$ fiber. Error bars denote the standard deviation of four samples. The penetration of the fluorescent spheres inside porcine clots was measured by Girsky et al.. ${ }^{7}$

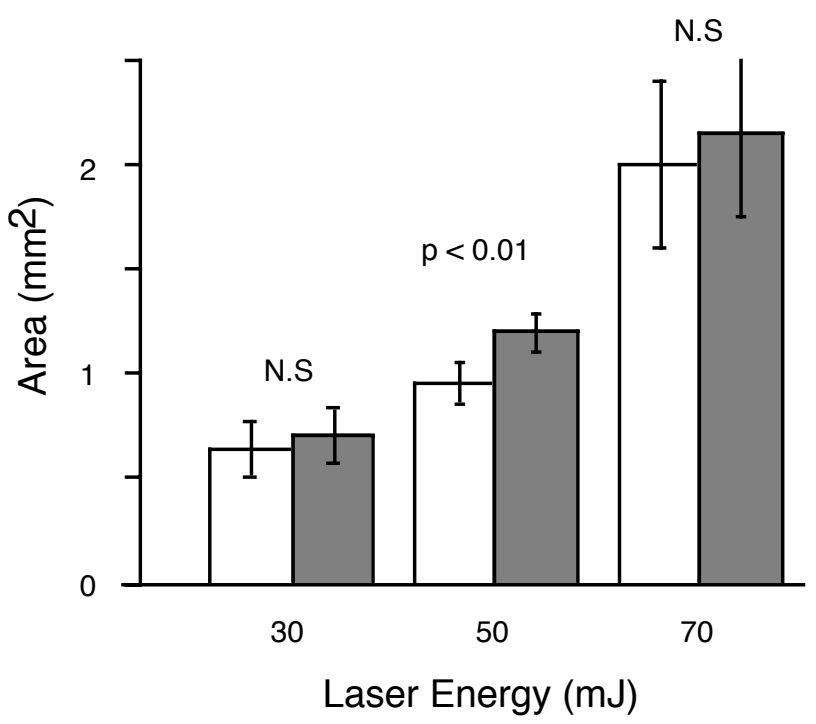

Figure 5. Areas including lumen areas created by laser ablation and stained areas due to the drug delivery as a function of laser energy by use of photomechanical drug delivery. Ninety laser pulses were delivered via a flushing catheter with a $300 \mu \mathrm{m}$ fiber onto the gelatin samples with an absorption coefficient of $100 \mathrm{~cm}^{-1}$. The open markers represent the lumen area and the filled markers indicate the areas including the lumen area and the stained area. Error bars present the standard deviation of four samples. 


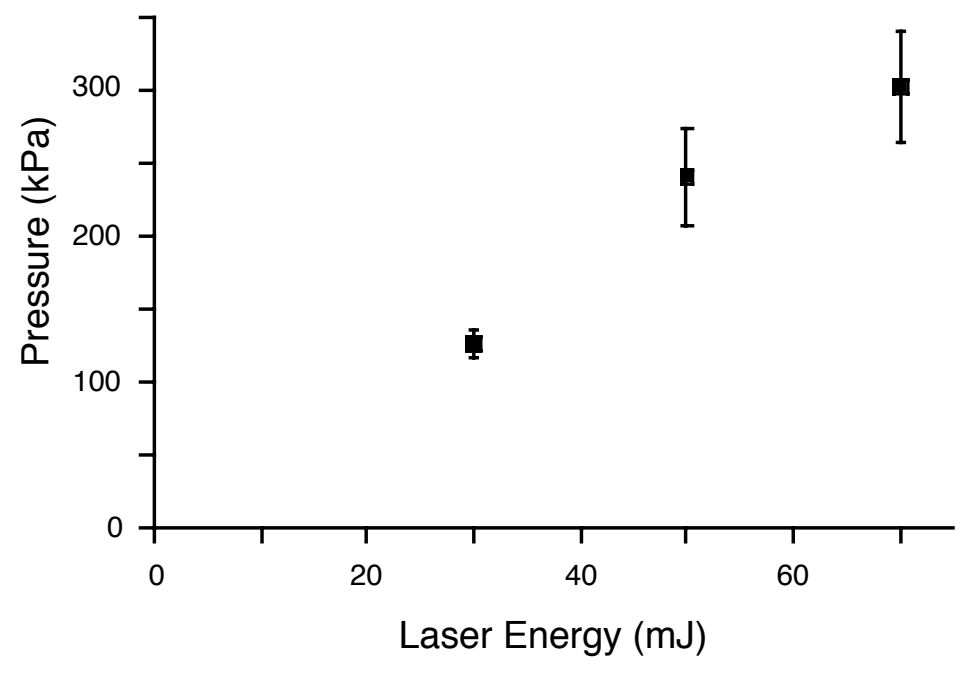

Figure 6. Bubble expansion pressure as a function of laser energy. The pressure signals were detected with a PVDF transducer placed $16 \mathrm{~mm}$ away from the ablation site under water. The absorbing gelatin samples had an absorption coefficient of $100 \mathrm{~cm}^{-1}$.

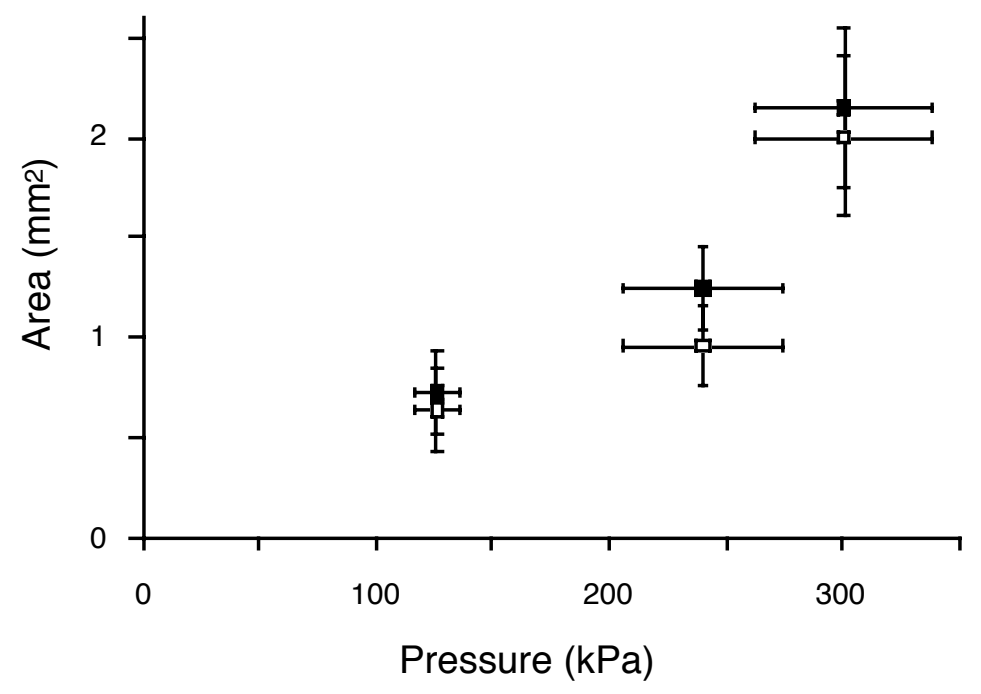

Figure 7. The areas produced by laser ablation (open squares) and drug delivery (filled squares) as a function of bubble expansion pressure on gelatin samples (Figure 6). 

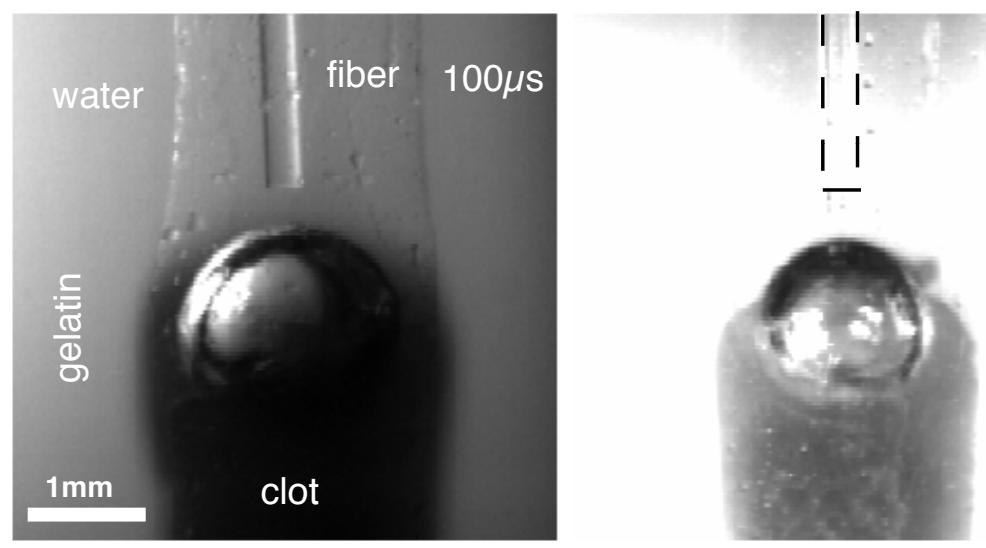

Figure 8. A laser-induced cavitation bubble formed in the clot. The right photo was taken to show the spherical shape of the bubble on the left.
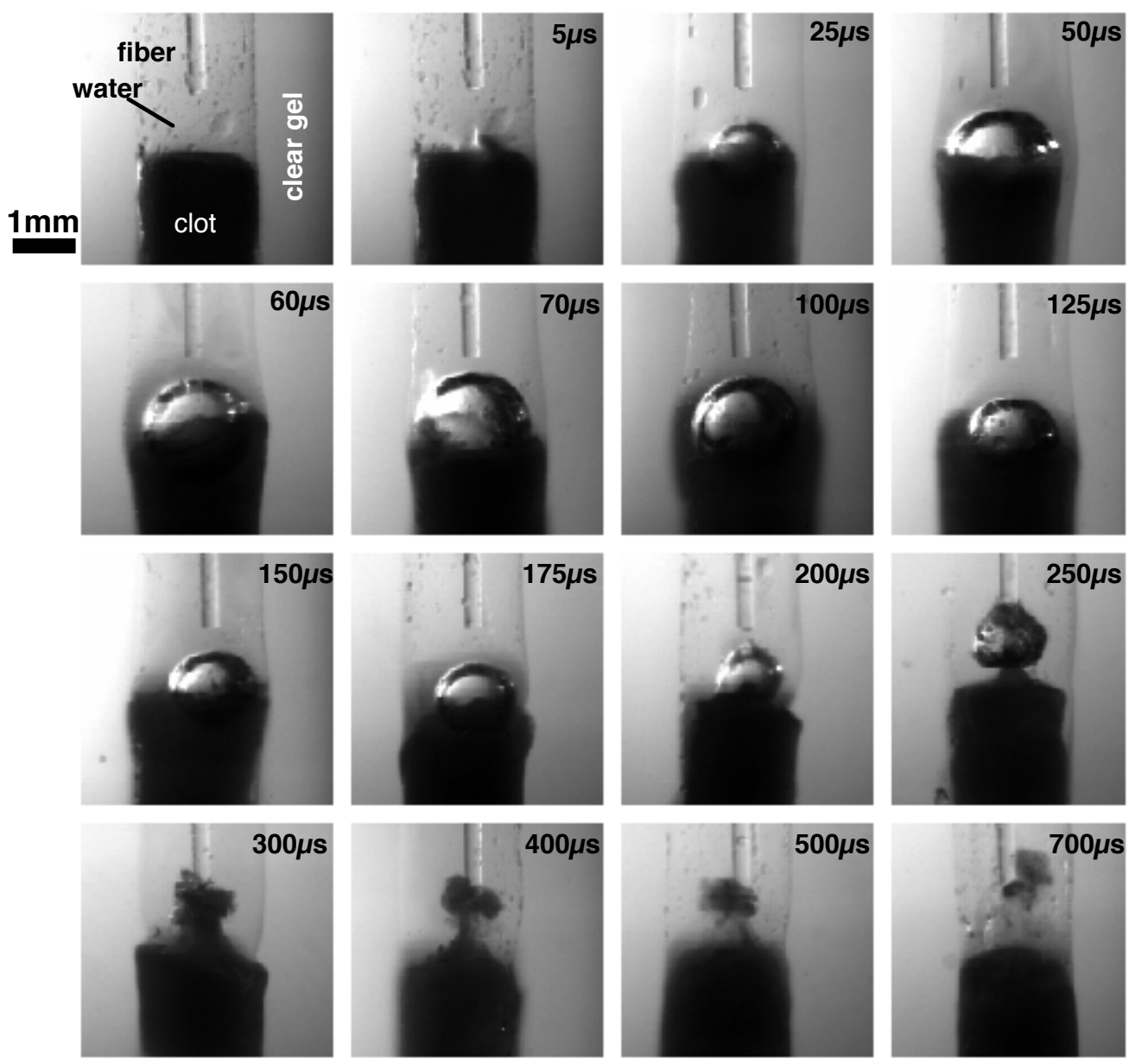

Figure 9. A series of flash photographs of cavitation bubble growth and collapse after the laser pulse. Single pulses of $50 \mathrm{~mJ}$ were delivered onto the clot via a $300 \mu \mathrm{m}$ fiber. The clot was confined in a gelatin sample with a cylindrical channel at center. The fiber was placed $1 \mathrm{~mm}$ above the clot under water. 


\section{DISCUSSION}

The results presented in this study demonstrate the possibility of using photomechanical drug delivery to enhance the thrombolysis process by delivering drug into thrombus during the laser thrombolysis procedure. Evidently, the lumen areas could be increased by delivering drug into the thrombus during laser ablation. For example, the mean of fluorescent sphere area and the maximal penetration depth were greater in the gelatin samples with photomechanical drug delivery: $0.24 \pm 0.05 \mathrm{~mm}^{2}$ for photomechanical drug delivery vs. $0.06 \pm 0.01 \mathrm{~mm}^{2}(p=0.005)$ for laser thrombolysis and $250 \pm 60 \mu \mathrm{m}$ for photomechanical drug delivery vs. $46 \pm 16 \mu \mathrm{m}(p<0.001)$ for laser thrombolysis. A similar result was reported by Girsky et $a .^{7}$ for photomechanical drug delivery to porcine clot confined in carotid arteries. Similar parameters such as the laser energy, fiber size, and fluorescent microsphere solution were used for their experiments. The mean of fluorescent sphere area was: $0.13 \pm 0.02 \mathrm{~mm}^{2}$ for photomechanical drug delivery vs. $0.02 \pm 0.004 \mathrm{~mm}^{2}$ ( $\left.p=0.01\right)$ for laser thrombolysis. The maximal penetration depth was $330 \pm 40 \mu \mathrm{m}$ for photomechanical drug delivery vs. $160 \pm 20 \mu \mathrm{m}(p=0.002)$ for laser thrombolysis.

Flash photographs showed that cavitation bubbles were formed after the laser pulses interacted with the clot. The growth and collapse of the cavitation bubbles caused dilation and consequent invagination of the channel wall of the gelatin samples. The flow motion may generate the hydrodynamic pressure that could force drug into the thrombus. Thrombus ejection was observed following the collapse of the cavitation bubble.

It is evident that the lumen areas increase with increasing laser energy. The higher bubble expansion pressures result in larger lumen areas, which agrees with an ablation efficiency study. ${ }^{8}$ However, the penetration depth of the spheres in gelatin samples was not simply proportional to the laser energy. The penetration increased at least before the laser energy reached $50 \mathrm{~mJ}$, and started deceasing somewhere between 50-70 mJ. One possible explanation is that two processes, mass removal and drug delivery, occur simultaneously during photomechanical drug delivery. More mass is removed although the drug can be driven into targets more deeply with higher pressures. ${ }^{4}$ We postulate that there is a threshold effect. The maximum penetration can be achieved when the pressure is below the threshold, since the pressures can only remove a certain amount of the gelatin, so that more stained areas are left. At suprathreshold energy the ablation process becomes more explosive, so that more mass is blew off, even though the spheres are driven more deeply into the gelatin if the penetration depth is measured from the center of the lumen rather than from the channel wall to the spheres. In general, the use of photomechanical drug delivery for enhancement of laser thrombolysis seems to be a trade-off between the mass removal by laser ablation and that by thrombolytic action.

In conclusion this study demonstrated that the thrombolysis process can be enhanced by delivering drug into thrombus during laser ablation. The lumen areas increased up to $25 \%$ if we consider the areas that may potentially

be dissolved by the drugs. The penetration depths of the spheres in the gelatin samples were not correlated with the laser energy, but the lumen sizes increased with increasing energy. The lumen areas created by laser ablation were correlated with the bubble expansion pressures, and the pressures were proportional to the laser energy.

\section{ACKNOWLEDGEMENTS}

We thank A. Shearin for his generous support provided to this project. This research was supported in part by the Murdock Foundation, Portland, Oregon and the Whitaker Foundation, Washington, DC.

\section{REFERENCES}

1. K. Gregory, "Laser Thrombolysis," in Interventional Cardiology, E. J. Topol, ed., vol. 2, ch. 53, pp. 892-902, W. B. Saunders Company, 1994.

2. E. A. Chasteney, P. S. Ravichandran, D. T. Bahlman, A. Shearin, and K. W. Gregory, "Rapid thrombolysis in experimental coronary artery bypass grafts," J. Am. Coll. Cardiol. 24 (suppl. A), p. 348A, 1995 (abstract).

3. J. F. Mitchel, D. B. Fram, F. D. P. II, R. Foster, J. A. Hirst, M. A. Azrin, L. M. Bow, A. M. Eldin, D. D. Waters, and R. G. McKay, "Enhanced intracoronary thrombolysis with urokinase using a novel, local drug delivery system: in vitro, in vivo, and clinical studies," Circulation 91, pp. 785-793, 1995.

4. H. Shangguan, L. W. Casperson, A. Shearin, K. W. Gregory, and S. A. Prahl, "Drug delivery with microsecond laser pulses into gelatin," Appl. Opt. 35, pp. 3347-3357, 1996. 
5. H. Shangguan, L. W. Casperson, A. Shearin, and S. A. Prahl, "Investigation of cavitation bubble dynamics using particle image velocimetry: implications for photoacoustic drug delivery," in SPIE Proceedings of Lasers in Surgery: Advanced Characterization, Therapeutics, and Systems VI, R. R. Anderson and A. Katzir, eds., vol. 2671, pp. 104-115, 1996.

6. H. Shangguan, Local Drug Delivery with Microsecond Laser Pulses: In vitro Studies. PhD thesis, Portland State University, 1996.

7. M. J. Girsky, K. W. Gregory, A. S. Shearin, and S. A. Prahl, "Photoacoustic drug delivery to arterial thrombus — a new method for local drug delivery," Circulation 94 (Suppl. I), pp. I-201, 1996 (abstract).

8. H. Shangguan, L. W. Casperson, and S. A. Prahl, "Microsecond laser ablation of thrombus and gelatin under clear liquids: Contact vs non-contact," IEEE J. Selected Topics Quantum Electron., 1997 (in press). 